\title{
Aplicação de um Processo Ágil para Implantação de Processos de Software baseado em Scrum na Chemtech
}

\author{
Alex Salgado ${ }^{1}$, Taciana Melcop ${ }^{1}$, Juliana Acchar ${ }^{1}$, Paulo Armando Rego ${ }^{1}$, \\ Analia Irigoyen Ferreiro Ferreira ${ }^{2}$, Anne Elise Katsurayama ${ }^{2}$, \\ Mariano Montoni ${ }^{2}$, David Zanetti ${ }^{2}$ \\ ${ }^{1}$ Chemtech - A Siemens Company \\ Rua da Quitanda, 50, $21^{\circ}$ andar, Centro, 20011-030. \\ Rio de Janeiro, RJ, Brasil. \\ \{alex.salgado, taciana.melo, juliana.acchar, paulo.rego\}@chemtech.com.br \\ ${ }^{2}$ ProMove - Business Intelligence Solutions \\ Rua Marechal Mascarenhas de Morais, 120, Copacabana, 22030-040 \\ Rio de Janeiro, RJ, Brasil. \\ \{analia, anneelisek,mmontoni, dzanetti\} @promovesolucoes.com
}

\begin{abstract}
This work presents the experience in implementing software processes adherent to CMMI-DEV Maturity Level 3 and MPS.BR Maturity Level $C$ in Chemtech, using an agile approach based on Scrum. The activities executed for implementing the processes are described in this work. Moreover, the lessons learned, success factors, difficulties and weaknesses observed during this experience are also described. The acquired knowledge during the execution of the process not only provided the means for the Chemtech's members to conduct the trainings in the defined processes, but also enabled the execution of pilot projects after eight sprints and four months since the begging of the implementation.
\end{abstract}

Resumo. Este trabalho apresenta a experiência na implantação de processos de software aderentes ao nível 3 do CMMI-DEV e ao nível C do MPS.BR na Chemtech, utilizando uma abordagem ágil baseada em Scrum. Além de detalhar as atividades utilizadas para esta implantação, este trabalho destaca as lições aprendidas, os fatores de sucesso, as dificuldades e os pontos fracos desta experiência. O conhecimento adquirido ao longo deste processo permitiu que os próprios colaboradores pudessem ministrar os treinamentos nos processos definidos, além de conduzir a execução dos pilotos após oito sprints e quatro meses desde o início da implementação.

\section{Introdução}

No Brasil, as organizações de desenvolvimento de software têm buscado, cada vez mais, a implantação de processos de software com o objetivo de melhorar a qualidade e reduzir os custos (Ferreira et al, 2006; Ferreira et al, 2007). Além disso, o mercado tem enxergado nestes certificados de qualidade uma forma de qualificar e diferenciar seus potenciais fornecedores. 
Neste sentido, a importância em implantar processos aderentes a normas e modelos de referência como o MPS.BR (Softex, 2009), o CMMI-DEV (Chrissis et al, 2006) ou a ISO 9001:2008 (ISO 9001, 2008) e a agilidade nesta implantação e na melhoria dos processos se torna, então, essencial para tornar as organizações de software mais competitivas e motivadas em manter a busca pela qualidade. Desta forma, assim como um processo de desenvolvimento de software, o trabalho de implantação de processos tem também como objetivo principal desenvolver produtos de alta qualidade no menor tempo possível.

Este artigo tem por objetivo relatar a experiência da Chemtech, iniciada em julho de 2009, na implantação de novos processos aderentes ao nível 3 do CMMI-DEV e ao nível $\mathrm{C}$ do MPS.BR, visando melhorar a qualidade dos processos implantados. Além deste objetivo, a organização tinha como metas: defini-los compatíveis com a cultura da organização (ágeis e facilmente adaptados às situações de inovação), integrá-los aos já definidos para a ISO 9001:2008 e que os próprios colaboradores fossem responsáveis pelos processos definidos, caracterizando assim a importância na Gestão do Conhecimento ao longo deste processo.

O desafio de alcançar o objetivo e as metas estabelecidas motivou a decisão da Chemtech por experimentar a agilidade também na implantação de processos de software.

Adicionalmente, algumas características da agilidade motivaram a definição deste processo na Chemtech, tais como (Kniberg, 2007): todos são um único time e estão comprometidos com o projeto (incluindo a equipe de consultoria neste contexto); a equipe deve entregar algo material, neste caso, parte da biblioteca de ativos (processos, componente de processo, seleção e implantação dos processos em ferramentas, diretrizes, guias, instruções técnicas etc) ao fim de cada iteração; iterações curtas e com espaço de tempo definido; adaptação do processo em situações específicas e o aprendizado contínuo.

De forma mais detalhada, neste artigo são apresentadas informações referentes a este processo de implantação de processos, suas dificuldades, fatores de sucesso e lições aprendidas. Na seção 2, é realizada uma breve revisão da literatura sobre métodos ágeis em gerenciamento de projetos. Detalhes da versão atual da abordagem utilizada na Chemtech baseada no Scrum estão descritos na seção 3. Na seção 4, são discutidas as lições aprendidas, dificuldades e resultados deste processo. Por fim, a seção 5 apresenta as considerações finais.

\section{Métodos Ágeis de Gerenciamento de Projetos}

Os métodos ágeis de desenvolvimento de software tiveram o seu início nos anos 80 , como parte de um movimento contra os métodos burocráticos de desenvolvimento. A criação desses métodos foi motivada pela necessidade de superar os problemas dos métodos tradicionais como o modelo cascata, considerado, pelos precursores dos métodos ágeis, lento e contraditório à maneira eficiente de trabalho dos engenheiros de software (Kniberg, 2007). Atualmente, destacam-se entre os métodos ágeis de desenvolvimento de software o XP (eXtreme Programming) e o Scrum. Enquanto o foco do XP está nas práticas de programação, o Scrum está focado nas práticas de gerenciamento de projetos. 
Inicialmente, o Scrum foi concebido como um estilo de gerenciamento de projetos em empresas de fabricação de automóveis e produtos de consumo (Nonaka e Takeuchi, 1986). Baseado na formação do Scrum do Rugby, estes autores notaram que projetos que utilizavam equipes pequenas e multidisciplinares (cross-functional) produziam os melhores resultados. Em 1993, Jeff Sutherland, John Scumniotales e Jeff McKenna documentaram e implementaram o Scrum na empresa Easel Corporation (Sutherland, 2004) a partir do estilo de gerenciamento de projetos observado por Nonaka e Takeuchi (1986). Finalmente, em 1995, Ken Schwaber formalizou a definição do Scrum para projetos de desenvolvimento de software e auxiliou na implantação deste método em organizações de desenvolvimento de software em todo o mundo (Schwaber, 2004; Schwaber, 2002).

Apesar de ser voltado para o gerenciamento de projetos de desenvolvimento de software, o Scrum pode ser aplicado em qualquer contexto no qual um grupo de pessoas necessite trabalhar juntas para atingir um objetivo comum como, por exemplo, um projeto de pesquisa. Além disso, o Scrum também pode ser aplicado como uma abordagem para gerenciamento de portfólio de projetos (Scrum de Scrums) (Schwaber, 2004)

As principais características do Scrum são (Schwaber, 2002):

- As fases do Scrum são divididas em sprints, ou seja, iterações que equivalem ao período de 2 a 4 semanas de trabalho da equipe. Cada sprint segue o ciclo PDCA (Plan-Do-Check-Act) e entrega um incremento de software pronto, o esforço de cada sprint está em se entregar itens com o maior valor de negócio (e prioridade) ao cliente, dando a ele algo real e de valor para o negócio.

- O conjunto de requisitos é denominado product backlog, e é priorizado pelo Product Owner (Cliente).

- Há uma breve reunião diária, denominada daily scrum meeting ou standup meeting, onde cada membro da equipe comenta sobre o progresso alcançado na tarefa, o trabalho a ser realizado e o que impede de seguir a diante com esta tarefa.

- O planejamento é breve e consiste na seleção dos itens do backlog para determinado sprint. Estas reuniões de planejamento são conhecidas como: Sprint Planning 1 (Planejamento do Sprint 1), Estimation Meeting (Reunião de Estimativa) ou Planning Poker e Sprint Planning 2 (Planejamento do Sprint 2).

- Entre os sprints há uma retrospectiva onde os membros da equipe refletem sobre o sprint passado. Estas reuniões são conhecidas como Sprint Review (Revisão do Sprint) e Sprint Retrospective (Retrospectiva do Sprint).

- O Scrum Master exerce o papel do facilitador que deve remover os obstáculos para que a equipe atinja o objetivo do sprint. Ele atua como um mediador, motiva a equipe, além de assegurar que as práticas do Scrum estão sendo seguidas.

No contexto deste trabalho, o framework de gerenciamento ágil Scrum foi selecionado como base para definir e implantar os processos de software aderentes ao nível 3 do CMMI-DEV e ao nível C do MPS.BR na Chemtech, conforme apresentado com mais detalhes na próxima seção. 


\section{O Processo Ágil para Implantação de Processos de Software na Chemtech}

Para realizar a implementação de melhorias nos processos da Chemtech, foi utilizado um processo ágil baseado: (i) em práticas definidas no framework do Scrum (Kniberg, 2007); (ii) na área de processo Foco na Melhoria do Processo Organizacional (OPF) do CMMI-DEV (Chrissis et al., 2006) e no processo Avaliação e Melhoria do Processo Organizacional do MPS.BR (AMP) (Softex, 2009); (iii) nos objetivos, metas e recursos disponíveis durante o planejamento do projeto; e (iv) nas necessidades e problemas encontrados ao longo da execução dos sprints na Chemtech.

Para apoiar esta abordagem foi definido um processo contendo uma descrição detalhada das atividades e das tarefas propostas. A notação utilizada para a sua representação é a de (Aguiar, 2004). O processo definido, conforme apresentado na Figura 1, contem tarefas organizacionais e é composto pelos seguintes subprocessos: (i) Planejamento do Projeto; (ii) Planejamento do Sprint \#N; (iii) Execução do Sprint \#N; (iv) Encerramento do Sprint \#N; e (v) Encerramento do Projeto.

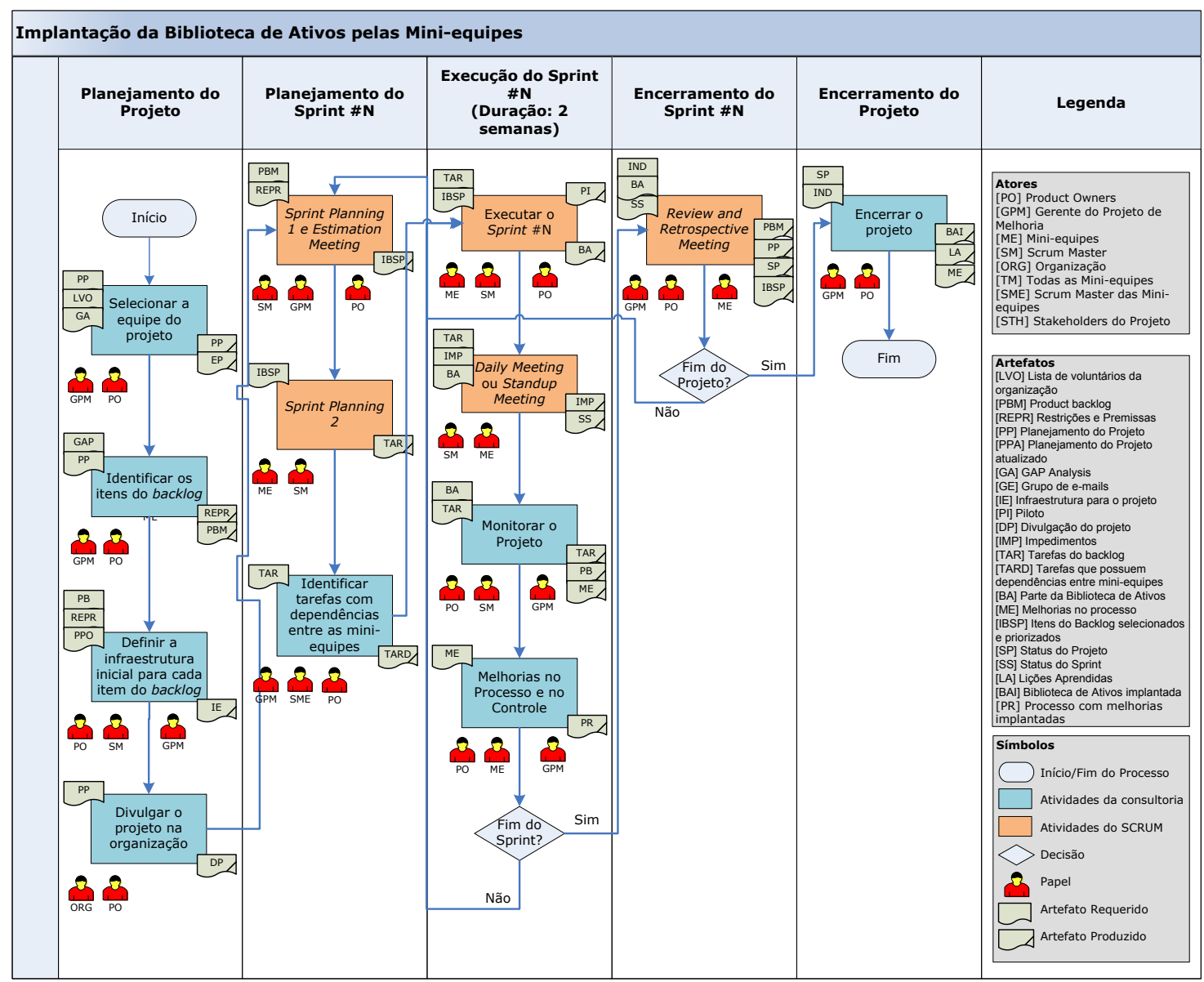

Figure 1. Processo Ágil para Implantação de Processos de Software

Foram definidos quatro importantes objetivos para o projeto de melhoria "Rumo ao CMMI 3", além da avaliação oficial que está planejada para Outubro de 2010. São eles: (i) definição dos processos aderentes à cultura da organização; (ii) integração dos processos já implantados e aderentes a ISO 9001:2008; (iii) disseminação do conhecimento; e (iv) validação dos processos definidos nos projetos pilotos. 
De forma mais detalhada, no Planejamento do Projeto de Melhoria, um item considerado essencial para o início de um Sprint na Chemtech foi a escolha das miniequipes que iriam compor a equipe do projeto. Foram definidas dez mini-equipes, que eram compostas por 3 colaboradores da empresa, segundo as seguintes áreas de interesse: Requisitos, Gerência de Projetos, Medição e Análise, Qualidade, Testes, Gerência de Portfólio de Projetos, Recursos Humanos, Compras, Arquitetura e Melhoria de Processos. As mini-equipes trabalhavam em paralelo e cada integrante da miniequipe tinha 20 horas dedicadas a cada Sprint de 2 semanas. A figura do Scrum Master era assumida por um dos membros da Chemtech, de cada mini-equipe enquanto o Product Owner era um membro da equipe de consultoria da ProMove.

Um Product Backlog foi definido e priorizado para cada mini-equipe com auxílio dos Products Owners. Além disso, o projeto de melhoria teve seus objetivos e equipe divulgados para todos os membros da organização. O primeiro Product Backlog baseava-se no estudo e discussões sobre as áreas de processo de cada mini-equipe. Os Products Backlog seguintes tratavam da definição de processos, guias, templates discussões e aprovações para as diversas áreas de processo.

Ao longo dos Sprints que duravam duas semanas, as tarefas eram detalhadas em post-its afixados em um quadro (Figura 2), e dúvidas eram esclarecidas pelos Product Owners. As tarefas que não eram finalizadas no atual Sprint retornavam para o Product Backlog e eram replanejadas pelo Product Owner e Scrum Master do projeto.

Nos cinco primeiros Sprints reuniões eram realizadas com o Scrum Master do projeto (Scrum de Scrums) e os Scrum Masters das mini-equipes, para o acompanhamento do projeto. Após o quinto Sprint estas reuniões envolviam todos os membros das mini-equipes disponíveis, principalmente pela necessidade de discussão dos pontos de integração das mini-equipes. Ao final de cada Sprint, um relatório de Status do Projeto era elaborado, com o objetivo de acompanhar o progresso de todas as mini-equipes envolvidas.

A fase de "Implantação da Biblioteca de Ativos" foi encerrada no Sprint \#8 e a fase atual é a de "Acompanhamento dos Processos nos Projetos".

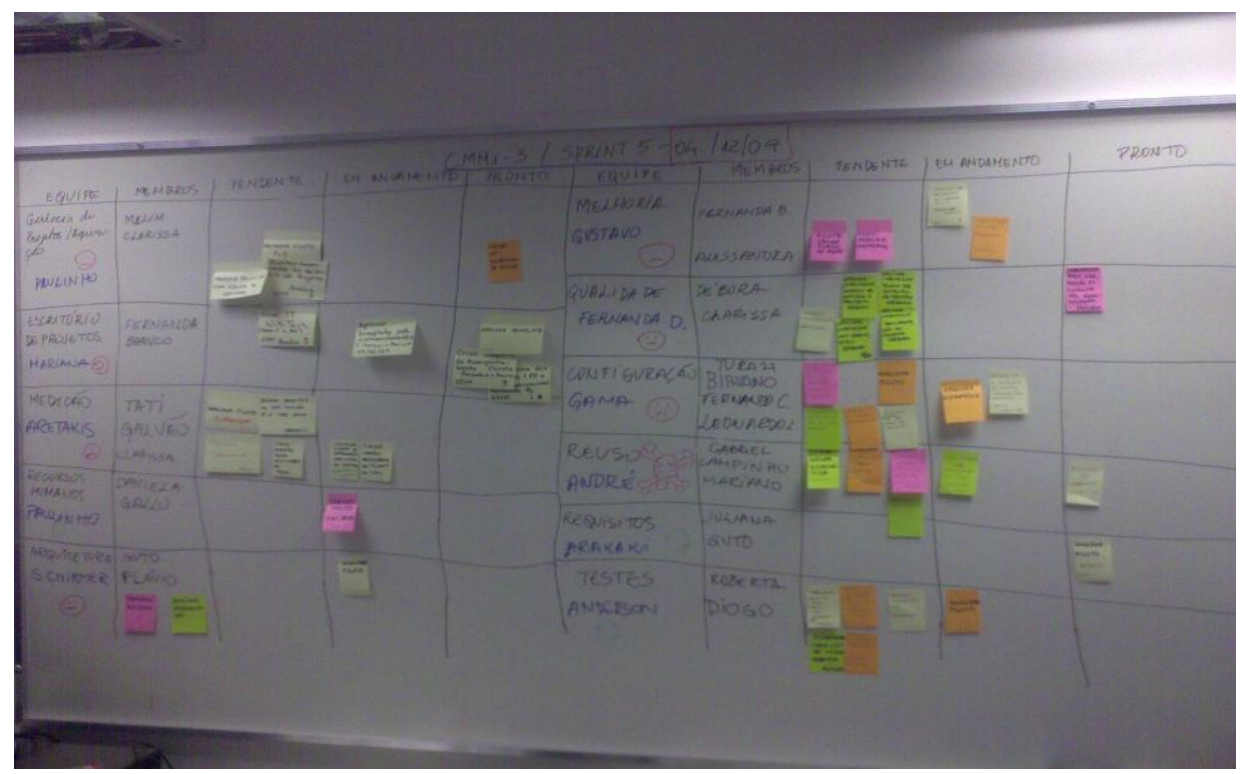

Figura 2. Quadro do SCRUM dividido por mini-equipe 


\section{Resultados e Lições Aprendidas}

Os resultados e lições aprendidas estão divididos conforme os objetivos do projeto de melhoria descritos no Capítulo 3. O objetivo relacionado à definição de processos aderentes à cultura da organização foi considerado satisfeito, visto que a biblioteca de ativos definida teve a participação e a aprovação efetiva de mais de trinta pessoas da organização (dividida em mini-equipes).

Com relação ao segundo objetivo, todos os processos definidos pela ISO 9001:2008, que estavam no escopo da implementação, foram considerados durante a definição de processos, conforme apresentado na Figura 3 e na Tabela 1. Poucas alterações pontuais foram realizadas. A Tabela 1 mostra os resultados quantitativos da Biblioteca de Ativos, enquanto a Figura 3 apresenta a estrutura de processos, com as respectivas inclusões, alterações e processos existentes que não tiveram modificações.

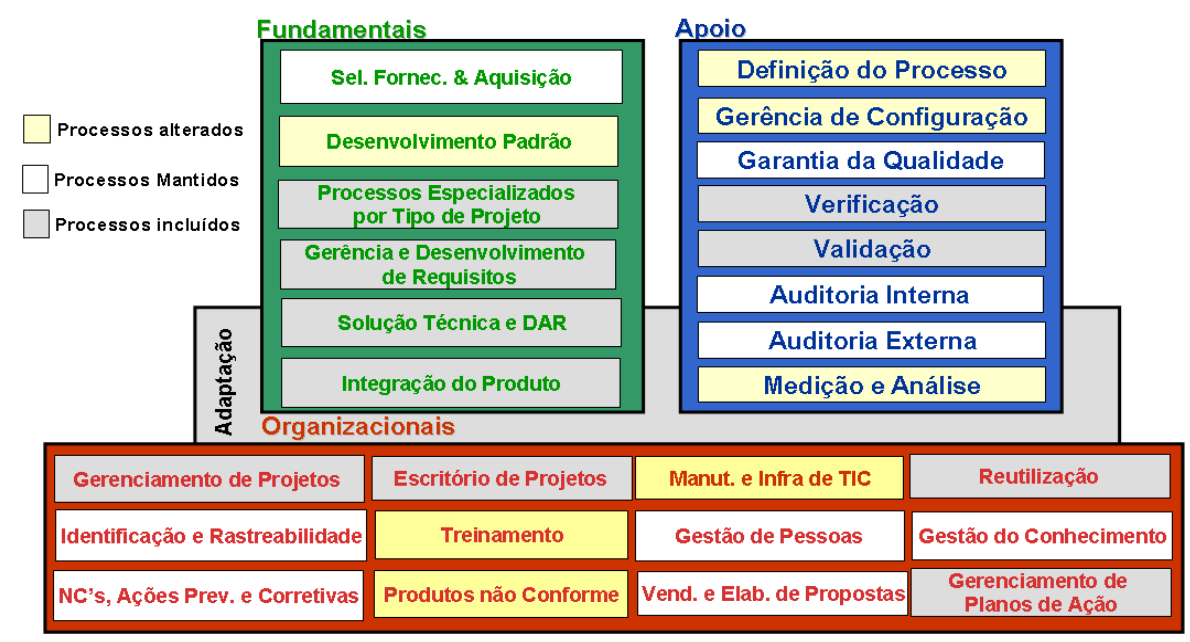

Figura 3. Estrutura após a definição da biblioteca de ativos

Tabela 1. Resultados quantitativos da biblioteca de ativos

\begin{tabular}{|c|c|c|c|c|c|c|c|}
\hline $\begin{array}{c}\text { Total de } \\
\text { documento } \\
\text { s } \\
\text { elaborados } \\
\text { para o } \\
\text { CMMi 3 }\end{array}$ & $\begin{array}{c}\text { Total de } \\
\text { processos } \\
\text { implantados } \\
\text { em feramentas }\end{array}$ & $\begin{array}{c}\text { Total de } \\
\text { documentos } \\
\text { ISO } \\
\text { incluídos no } \\
\text { escopo }\end{array}$ & $\begin{array}{c}\text { Total de } \\
\text { documento } \\
\text { s ISO } \\
\text { alterados }\end{array}$ & $\begin{array}{c}\text { Total de } \\
\text { envolvidos } \\
\text { no Projeto }\end{array}$ & $\begin{array}{c}\text { Total de } \\
\text { ferramentas } \\
\text { utilizadas } \\
\text { no ambiente } \\
\text { padrão }\end{array}$ & $\begin{array}{c}\text { Total de } \\
\text { material de } \\
\text { treinamento } \\
\text { elaborado }\end{array}$ & $\begin{array}{c}\text { Total de } \\
\text { Pilotos } \\
\text { Realizados }\end{array}$ \\
\hline 77 & 11 & 36 & 7 & 35 & 03 & 20 & 8 \\
\hline
\end{tabular}

O terceiro objetivo alcançado foi a gestão de conhecimento ao longo do processo, ou seja, a disseminação efetiva do conhecimento sobre os modelos de referências adotados e os próprios processos da organização. Uma evidência disso é que os próprios colaboradores da organização integrantes das mini-equipes participaram da elaboração e ministraram os treinamentos para os demais membros da organização.

Com relação ao quarto objetivo, ao final do Sprint \#8, tínhamos 8 dos 10 projetos pilotos (execução dos processos definidos pelas mini-equipes em projeto reais) executados. Foram solicitadas melhorias na Biblioteca de Ativos inicialmente definida e os processos foram considerados "prontos" para serem institucionalizados. 
Dificuldades de planejamento e monitoração dos sprints foram observadas ao longo do projeto, muitas delas devido ao compartilhamento de recursos com os projetos da organização. As principais dificuldades foram: (i) as retrospectivas eram feitas ao longo dos sprints durante as reuniões de acompanhamento, desviando o foco para a melhoria no processo definido e não no cumprimento dos objetivos dos sprints, tornando as reuniões demoradas; (ii) nem todos os integrantes das mini-equipes participavam das reuniões de acompanhamento, dificultando a comunicação e a visibilidade da situação do sprint; (iii) a dificuldade em definir o tamanho e o tempo necessário para executar uma determinada atividade, causando atrasos no projeto; e (iv) alta rotatividade dos integrantes da mini-equipe.

Por conta da inexperiência dos membros da equipe em definir processos e exercer o papel de Scrum Master, a consultoria foi uma presença constante, orientando e avaliando os artefatos gerados pelos membros das mini-equipes. Caso esta presença não fosse constante, a visibilidade do andamento dos projetos poderia ser prejudicada. Mesmo com essas dificuldades, os resultados obtidos ao final da implantação da Biblioteca de Ativos foram positivos, motivando a organização a continuar utilizando o processo definido, com algumas adaptações, para a próxima fase do projeto que envolve o acompanhamento dos processos na organização.

Os indicadores de progresso do projeto estão ligados ao percentual de aderência as práticas de cada área de processo, ou seja, o acompanhamento dos projetos será realizado com o preenchimento da planilha de indicadores, indicando o momento adequado para a realização do SCAMPI - C.

\section{Conclusão}

A utilização de métodos ágeis para implantação de melhorias mostrou-se eficiente principalmente pela disseminação do conhecimento sobre os modelos de referência e boas práticas que permitiu que os membros das mini-equipes tivessem uma visão crítica das áreas de processo, tornando o processo definido mais adequado à cultura e as características da organização. Além disso, a equipe ficou altamente motivada com a dinâmica adotada.

Outro importante ganho na utilização da agilidade na implantação de processos foi que, ao utilizar incrementos e focar na melhoria contínua, a aderência do processo atual aos novos processos foi antecipada e consequentemente facilitada.

Porém, é importante destacar alguns aspectos considerados críticos para o sucesso do projeto de melhoria como: os membros da organização devem gostar de definir processos e ferramentas; as mini-equipes devem ser preferencialmente compostas com perfis heterogêneos; a consultoria, como Product Owner, deve estar accessível e presente sempre que necessário; trocar os membros das mini-equipes sempre que existirem conflito de prioridades com outros projetos; e identificar melhorias no controle do projeto sempre que necessário. 
Os esforços no acompanhamento dos processos serão continuados até a avaliação da implementação dos processos na organização onde mais lições aprendidas serão coletadas e melhorias no processo definido para este projeto serão realizadas, permitindo obter mais evidências dos potenciais benefícios da utilização do Scrum para agilizar a implantação de processos de software.

\section{Referências}

ABNT NBR ISO 9001:2008, Sistema de Gestão da Qualidade - Requisitos, Associação Brasileira de Normas Técnicas, v. ISO 9001:2008.

Aguiar, H. V., 2004, PEPP: Processo de Software para Empresas de Pequeno Porte Baseado no Modelo CMMI, Graduação, Departamento de Ciência da Computação, Universidade Federal de Lavras.

Chrissis, M. B., Konrad, M., Shrum, S., 2006, CMMI (Second Edition): Guidelines for Process Integration and Product Improvement, Addison Wesley Professional.

Ferreira, A. I. F., Cerqueira, R., Santos, G., et al., 2006, "ISO 9001:2000, MPS.BR Nível F e CMMI Nível 3: Uma Estratégia de Melhoria de Processos na BL Informática", V SBQS, pp. 375-382, Vila Velha, Brasil.

Ferreira, A. I. F., Santos, G., Cerqueira, R., et al., 2007, "Applying ISO 9001:2000, MPS.BR and CMMI to Achieve Software Process Maturity: BL Informatica's Pathway", 29th ICSE, Minneapolis, Estados Unidos.

Kniberg, H., 2007, “Scrum e XP direto das Trincheiras”, Editora C4 Media, Publisher of InfoQ.com.

Nonaka, I., Takeuchi, H., 1986, The new product development game, Harvard Business Review.

Schwaber, K., 2004, Agile Project Management with Scrum, Microsoft Press.

Schwaber, K., Beedle, M., 2002, Agile Software Development with Scrum, Prentice Hall.

Softex, 2009, "MPS.BR: Melhoria de Processo do Software Brasileiro", Guia Geral, Disponível em: http://www.softex.br/mpsbr.

Sutherland, J., 2004, Agile Development: Lessons Learned from the First Scrum, Cutter Agile Project Management Advisory Service: Executive Update, volume 05, pp. 0104. 